\title{
Existence of Stationary Equilibrium in the Markets for New and Used Durable Goods*
}

\author{
Hideo Konishi ${ }^{\dagger} \quad$ Michael T. Sandfort ${ }^{\ddagger}$
}

January 28, 2000

\begin{abstract}
We prove the existence of stationary equilibrium in the primary and secondhand markets for an indivisible consumer durable in a general model with stochastic degradation and endogenous scrappage decisions. Unlike Rust (1985), we introduce transaction costs in the model as a motivation for consumer holdings of durables across multiple quality levels. In addition, we allow for multiple types of durables (e.g., Porsche and $\mathrm{BMW}$ ). Since we use a fixed point theorem in making the existence argument, we do not need to invoke the single-crossing property on consumer tastes.
\end{abstract}

Keywords: Durable goods, Transaction costs, Existence of stationary equilibrium JEL Classification: C62; D11; D41

*The authors thank Anna Bogomolnaia, Jim Dolmas and Greg Huffman for valuable comments and take responsibility for all remaining errors.

${ }^{\dagger}$ Department of Economics, Boston College, Chestnut Hill, MA 02467-3806, USA.

${ }^{\ddagger}$ Corresponding author. Department of Economics, Southern Methodist University, Dallas, TX 75275 0496, USA. Phone - 214-768-3856. Email - sandfort@post.smu.edu. 


\section{Introduction}

Durables are consumed over many periods, so they can be (and often are) traded as used goods. For a variety of reasons, one of the most challenging aspects of modeling durables is appropriately modeling this secondhand market. First, if durables degrade over time, the secondhand market contains goods of a variety of different qualities, each imperfectly substitutable for a brand new durable. The model of the secondhand market must, therefore, account for these (vertically) differentiated products. Second, the extent of the aforementioned product spectrum should clearly depend on the willingness of consumers to hold very degraded durables, i.e., scrappage should be endogenous.

Rust (1985) is the first paper to present a model with the above two characteristics. He both proves the existence of a stationary equilibrium of the model and, for a particular specification of the depreciation process, characterizes equilibrium prices. Unfortunately, his model leaves unexplained one significant characteristic of consumers' behavior, the holding of durables across multiple periods. To the contrary, in his model every consumer holds a durable (e.g., car) essentially only one period, repeatedly purchasing the same grade car.

This is apparently an undesirable feature of the Rust model, probably attributable to the fact that the Rust economy is too perfect to describe the real world. In the absence of some sort of market frictions or imperfections, consumers have no incentive to hold their durables across multiple quality levels. Each heterogeneous consumer will choose a durable from the product spectrum so as to maximize her net surplus. One way to explain consumers' car holding behavior is to introduce asymmetric information between sellers and buyers. Akerlof (1970) argues that asymmetric information between buyers and sellers of used durables may entirely shut down the market in secondhand durables. Recent work by Hendel and Lizzeri $(1997,1999)$, however, suggests some concerns about the pre-trade allocation of durables assumed by Akerlof. They show the existence of a market equilibrium in a model with durables of two vintages ("new" and "used") and exogenous scrappage (i.e., durables become useless after two periods). They argue that, while asymmetric information may attenuate equilibrium trade in the secondhand market, the used market never shuts down completely and the distortions produced by asymmetric information are smaller than previously thought.

There is another way to explain consumer holdings of durable goods. If there are positive transaction costs in trade (e.g., search for replacement durables is costly), consumers may hold their durables over multiple periods to economize on transaction costs. Sandfort (1999b) presents a model in which every transaction is subject to a fixed, per-transaction, expenditure and in which durables degrade stochastically from one quality level to another. Thus, for any given durable, there are a variety of close, but imperfect, substitutes. Scrappage is endogenous, so the willingness of consumers to hold a durable across multiple quality levels is related to the costliness of transactions. Numerical examples show that, as the transaction cost increases, consumers tend to hold their durables over a wider range of qualities (e.g., on average, they hold their cars longer). As the transaction cost increases, the markets for high quality used durables close, since all owners of these durables prefer to hold the durable to a more degraded state rather than trade it and incur the transaction cost. In the limit, as 
the transaction cost becomes large, trade in used durables of every quality ceases.

In this paper, we present a model of the primary and secondhand markets for a nondivisible consumer durable with positive transaction costs. Time is discrete, and in every period consumers have the option to either continue with their current holdings (possibly none) of the durable or to replace their current holdings with the best available (possibly used) alternative. Durables are available in a variety of types (e.g., Porsche and BMW) and each type degrades according to a type-specific stochastic process. As a result, each consumer's choice problem reduces to optimal control of the stochastic processes governing depreciation. Consumers are heterogeneous in their tastes for type and degradation of the durable good, but we do not impose a single-crossing condition on tastes, as does Rust (1985).

Introducing transaction costs requires us to depart significantly from Rust's (1985) approach in proving existence of a stationary equilibrium. To explain the difference, we first briefly describe Rust's (1985) approach. Without transaction costs, one can show that each consumer always chooses to consume a durable of a single grade (i.e., level of degradation). Thus, it is easy to find a stationary distribution of durables over grades by setting a threshold grade such that durables of this grade or lower are scrapped, while durables of higher grades are not. Under the assumption that indifference curves satisfy the single-crossing property, consumers sort themselves over all grades higher than the threshold grade. Using this "location function" which describes consumer choices, Rust then finds a price function supporting these choices. Finally, the intermediate value theorem guarantees the existence of a threshold grade that implies, via the related stationary distribution and location function, a price function consistent with the exogenous prices of brand new cars and cars sold as scrap.

With transaction costs, consumers' behavior is no longer so simple, since consumers' decision rules are no longer characterized by replacement in every period. Thus, we can no longer separate the consumers' decision problem from that of obtaining supporting prices. Instead, we need a fixed point theorem (in particular, Fan-Glicksberg's fixed point theorem) to find a price vector and durable good distribution simultaneously. The proof is based on the method of Jovanovic and Rosenthal (1988) in finding a stationary equilibrium in an anonymous sequential game (see also Mas-Colell (1984)). One difficulty related to introducing prices into their model is ensuring that the price vector is bounded above. We show in our Lemma that the fixed point prices are equilibrium prices. Given that we use a fixed point theorem, introduction of multiple types of durables comes at no cost, as does relaxation of the single-crossing condition.

The remainder of this section provides a brief background on competitive models of durable goods. The second section presents the model, equilibrium concept, assumptions and our main result, the existence theorem. The third section presents some remarks on the assumptions sufficient to show the existence of equilibria of the model. The proof of our existence result may be found in the fourth section. 


\section{$1.1 \quad$ Literature Review}

Unsurprisingly, the literature modeling durable goods under competition is closely connected to the product differentiation literature and contains both discrete and continuous choice models. Bresnahan (1981) and Manski $(1982,1983)$ were the first to model automobile demand as a discrete choice problem, an approach extended by Berkovec (1985). The empirical studies in this tradition, including more recent work by Berry, Levinsohn and Pakes (1994), concentrate on the automobile industry because of both the importance of the industry and the availability of high quality registration data. Sandfort (1999a) considers a discrete choice model of automobile demand in which automobile holdings are motivated by the costliness of time spent in search for a replacement.

Models which employ a continuum of agents or a continuum of goods include Swan (1970), Liebowitz (1982), Bond (1983) and Rust (1985). The Rust model is the most general of these, since it assumes neither perfect rental markets in the durable nor exogenously-lived durables. As noted above, each consumer in Rust's model chooses an optimal location in the product spectrum based on her preference for quality (which is subject to a single crossing condition). Allowing the product spectrum to be endogenous then forms a very natural way of incorporating endogenous scrappage into the model. The key feature distinguishing this model from a standard product differentiation model is the constraint which the depreciation process places on the quantity of each good which can be supplied. Used goods cannot just be produced; they must depreciate from new goods. Hence, the product spectrum and prices must be computed in such a way that they are consistent with both consumer preferences and depreciation. None of the aforementioned models has very much to say about individual consumer holdings of durable goods.

Anderson and Ginsburgh (1994) consider the effect of transaction costs on the primary and secondhand markets for durables when durables depreciate deterministically from a "new" quality level to a "used" quality level. ${ }^{1}$ Using single-crossing of indifference curves, they characterize the relationship between the costliness of transactions and activity in the secondhand market. Our approach does not subsume that of Anderson and Ginsburgh, although we add variation in types of durables (e.g. BMW, Mercedes, Volvo) and endogenous scrappage to the model. ${ }^{2}$ Sandfort (1999c) considers a model in which durables may be one of two distinct types. Each type of durable depreciates through a continuum of possible states. In this context, one may address the substitutability of depreciable and non-depreciable components of an automobile (e.g., odometer reading versus wheelbase or maximum horsepower).

\footnotetext{
${ }^{1}$ Both Liebowitz (1982) and Bond (1983) consider depreciation of this kind.

${ }^{2}$ While our model does not treat the case of deterministic depreciation, the extension of our approach to include deterministic depreciation is straightforward.
} 


\section{The Model}

\subsection{Overview of Economy}

There are finite types of durable goods (BMW, Mercedes, Volvo, Porsche and so on). Each durable can occupy one of a variety of different conditions or "states" (e.g., odometer readings). There are periods $t=0,1,2, \ldots$ A physical stochastic process describes the transformation of a durable's condition in period $t$ to its condition in period $t+1$. There are atomless, infinitely-lived, consumers with heterogeneous tastes for the services provided by durables. That is, some like an old Porsche better than a brand new BMW, while some do not. Each consumer is assumed to consume at most one unit of a durable good. Since durables degrade over time, a given consumer will occasionally desire to replace her durable, either with a brand new durable or with a secondhand one. In our economy, there are complete markets for any type of durable in any state and consumers have perfect information about durables (so that there is no lemon problem). Prices of new and used durable goods are determined competitively. A stationary equilibrium requires that both prices and the distribution of durables are stationary.

\subsection{Commodities}

There are $J$ types of durable goods in the economy. The set of durable goods $\{1, \ldots, J\}$ is denoted by $\mathcal{J}$. There are $S+1$ states (levels of degradation) for each durable good, and the set of states $\{0,1, \ldots, S\}$ is denoted by $\mathcal{S}$. State $s=0$ indicates a brand new durable good, while increasing $s \in \mathcal{S}$ indicates increasing degradation. Thus, for every durable $j \in \mathcal{J}$, the state $S$ denotes a completely degraded durable (whose value, in equilibrium, should be no greater than scrap). It follows that there are $J(S+1)$ physically different commodities among which consumers may choose. Each commodity is identified by a pair $(j, s) \in \mathcal{J} \times \mathcal{S}$. For each type of durable good $j \in \mathcal{J}$, transition between states is governed by a Markovian depreciation process: $\phi_{j}: \mathcal{S} \times \mathcal{S} \rightarrow[0,1]$ such that $\sum_{s^{\prime} \in \mathcal{S}} \phi_{j}\left(s, s^{\prime}\right)=1$ for any $s \in \mathcal{S}$. The decision not to consume a durable is denoted by $\emptyset$, so that a consumer's durable good consumption in any period may be represented by $\omega \in \Omega \equiv(\mathcal{J} \times \mathcal{S}) \cup\{\emptyset\}$. In addition, there is a perfectly divisible good (money), which is treated as the numeraire.

\subsection{Commodity Markets}

We assume that supply of brand new durable goods is perfectly elastic, i.e., that, for each $j \in \mathcal{J}$, durables in state $s=0$ are perfectly elastically supplied at price $\bar{p}_{j}>0$. We assume further that durables of type $j \in \mathcal{J}$, regardless of state, may be sold as scrap. Thus, demand for scrap durables of type $j \in \mathcal{J}$ is also perfectly elastic at $\underline{p}_{j} \geq 0$. Naturally, we assume $\bar{p}_{j}>\underline{p}_{j}$. Since markets are complete, there is price for each durable at each state. Let $p_{j s}(t)$ be the price of durable $(j, s)$ at period $t$ and $p_{\emptyset}=0$ denote the price of not consuming a 
durable. The price set for all durable goods is denoted by $P \equiv \Re_{+}^{J(S+1)} \times\{0\} .^{3}$

\subsection{Consumers}

There is a continuum of consumers with different characteristics. The set of characteristics is denoted by a space $A$, and $a \in A$ is its representative element. Each consumer consumes at most one durable good at each period $t=0,1,2, \ldots$, denoted $\omega(t) \in \Omega$. Every consumer's utility function is time-additively separable, so the utility that a type $a$ consumer obtains from her consumption plan $(\omega(t), x(t))_{t=0}^{\infty}$, (where $x(t)$ denotes consumption of the divisible good at period $t$ ), is written as:

$$
\sum_{t=0}^{\infty}\left(\beta^{a}\right)^{t} U^{a}(\omega(t), x(t)),
$$

where $U^{a}: \Omega \times \Re_{+} \rightarrow \Re$ denotes atemporal utility from consuming a durable good and divisible money, and $\beta^{a} \in[0,1)$ denotes type $a$ consumer's discount factor. We assume that $U^{a}$ is quasi-linear in money in order to eliminate wealth effects:

$$
U^{a}(\omega(t), x(t))=u_{\omega(t)}^{a}+x(t) .
$$

Finally, each time a consumer of type $a \in A$ transacts in the market for durables, she pays a transaction cost $c^{a} \geq 0$. To concentrate on consumers' choices of durable goods, we follow Rust (1985) in modeling neither consumers' savings decisions nor their liquidity constraints. In addition, we assume that the interest rate is zero. Consequently, a consumer type in this durable goods economy is fully characterized by a triple $\left(\beta^{a}, c^{a},\left(u_{\omega}^{a}\right)_{\omega \in \Omega}\right)$. Thus, the space of consumer types, $A$, can be regarded as the space of utility functions, i.e., the metric space $A \subset[0,1) \times \Re_{+} \times \Re_{+}^{\# \Omega}$. ${ }^{4}$ Since we are interested in a stationary distribution of durables over consumers, it is convenient for us to use Hart, Hildenbrand, and Kohlberg's (1974) equilibrium-as-distribution approach. The distribution of consumers is described by a probabilistic measure $\nu$ on $A(\nu(A)=1)$.

\subsection{Timing of Events}

We now describe the timing of depreciation and trade. At the beginning of each period $t=1,2, \ldots$, each consumer owns a durable good $\omega \in \Omega$, which is the outcome of trade in the previous period, $t-1$ (or the initial allocation in the case $t=1$ ). Ownership of the durable $\emptyset \in \Omega$ is regarded as the choice not to consume a durable in period $t-1$. Immediately upon entering period $t$, a durable in state $\omega=(j, s)$ depreciates according to the exogenous depreciation process $\left\{\phi_{j}(s, \cdot)\right\}$ to a new state $\omega^{\prime}=\left(j, s^{\prime}\right)$. Of course, the durable $\emptyset$ does

\footnotetext{
${ }^{3}$ Of course, at an equilibrium, perfectly elastic supply for brand new durable goods and perfectly elastic demand for scraps will imply constraints on prices.

${ }^{4}$ Formally, the representative consumer is $a=\left(\beta, c,\left(u_{\omega}\right)_{\omega \in \Omega}\right) \in A$. For the purpose of readability, we abuse conventional notation, denoting $a$ 's transaction cost (for example) as $c^{a}$ rather than $a_{c}$.
} 
not depreciate. Taking the durable $\omega^{\prime}$ as her endowment, the consumer must then decide on her durable goods holdings over period $t$. She may continue to hold her current durable $\omega^{\prime}$ or may sell it on the secondhand market, purchasing a replacement $\omega^{\prime \prime}=\left(j^{\prime \prime}, s^{\prime \prime}\right) \in \Omega$. Production of brand new $(s=0)$ durable goods and the trade described above take place contemporaneously.

\subsection{Consumers' Choice Problem}

Since our focus is to find a stationary equilibrium allocation, we assume that the market price $p \in P$ is constant over time as well. Based on her (post-depreciation) endowment durable $\omega \in \Omega$ and on prevailing market prices $p$, the consumer decides whether to replace $\omega$ and determines an optimal replacement $\omega^{\prime} \in \Omega$. To formalize the consumer's choice problem, it is convenient to define the consumer's value function $V: A \times \Omega \times P \rightarrow \Re_{+}$such that:

(Case-I) If $\omega=(j, s) \in \mathcal{J} \times \mathcal{S}$,

$$
V(a, j, s, p)=\max \left\{\begin{array}{c}
u_{j s}^{a}+\beta^{a} \sum_{\tilde{s} \in \mathcal{S}} \phi_{j}(s, \tilde{s}) V(a, j, \tilde{s}, p), \\
\max _{\left(j^{\prime}, s^{\prime}\right) \in \mathcal{J} \times \mathcal{S}}\left(u_{j^{\prime} s^{\prime}}^{a}+p_{j s}-p_{j^{\prime} s^{\prime}}-c^{a}+\beta^{a} \sum_{\tilde{s} \in \mathcal{S}} \phi_{j^{\prime}}\left(s^{\prime}, \tilde{s}\right) V\left(a, j^{\prime}, \tilde{s}, p\right)\right), \\
p_{j s}-c^{a}+\beta^{a} V(a, \emptyset, p)
\end{array}\right\},
$$

(Case-II) If $\omega=\emptyset$,

$$
V(a, \emptyset, p)=\max \left\{\begin{array}{c}
\beta^{a} V(a, \emptyset, p), \\
\max _{\left(j^{\prime}, s^{\prime}\right) \in \mathcal{J} \times \mathcal{S}}\left(u_{j^{\prime} s^{\prime}}^{a}-p_{j^{\prime} s^{\prime}}-c^{a}+\beta^{a} \sum_{\tilde{s} \in \mathcal{S}} \phi_{j^{\prime}}\left(s^{\prime}, \tilde{s}\right) V\left(a, j^{\prime}, \tilde{s}, p\right)\right)
\end{array}\right\}
$$

since $p_{\emptyset}=0$ and $u_{\emptyset}^{a}$ is normalized to zero. In each of the above two cases, the first component of the contents of the brace gives the expected present value of keeping the endowment durable (i.e., not trading). The second component gives the expected present value of optimal replacement with a durable $\omega^{\prime}=\left(j^{\prime}, s^{\prime}\right)$, net of the transaction cost. Note that the optimal replacement does not depend on the endowment durable $\omega$. This is a result of the absence of wealth effects under quasi-linear utility and the absence of borrowing constraints. In Case-I, the third component gives the value of selling the endowment durable without replacement, again, net of the transaction cost.

Now consider a consumer of type $a$ endowed (before trade) with a durable in state $\omega$. We say that a durable good $\omega^{\prime}$ is her optimal choice iff

$$
\tilde{V}\left(a, \omega, \omega^{\prime}, p\right) \geq \tilde{V}(a, \omega, \Omega, p),
$$


where $\tilde{V}: A \times \Omega \times \Omega \times P \rightarrow \Re$ is such that

(Case-I) if $\omega^{\prime}=\omega=(j, s)$,

$$
\tilde{V}\left(a, \omega, \omega^{\prime}, p\right)=u_{j s}^{a}+\beta^{a} \sum_{\tilde{s} \in \mathcal{S}} \phi_{j}(s, \tilde{s}) V(a, j, \tilde{s}, p)
$$

(Case-II) if $\omega^{\prime}=\omega=\emptyset$,

$$
\tilde{V}\left(a, \omega, \omega^{\prime}, p\right)=\beta^{a} V(a, \emptyset, p),
$$

(Case-III) if $\omega^{\prime}=\left(j^{\prime}, s^{\prime}\right) \neq \omega$,

$$
\tilde{V}\left(a, \omega, \omega^{\prime}, p\right)=u_{j^{\prime} s^{\prime}}^{a}+p_{\omega}-p_{j^{\prime} s^{\prime}}-c^{a}+\beta^{a} \sum_{\tilde{s} \in \mathcal{S}} \phi_{j^{\prime}}\left(s^{\prime}, \tilde{s}\right) V\left(a, j^{\prime}, \tilde{s}, p\right),
$$

(Case-IV) if $\omega^{\prime}=\emptyset \neq \omega$,

$$
\tilde{V}\left(a, \omega, \omega^{\prime}, p\right)=p_{\omega}-c^{a}+\beta^{a} V(a, \emptyset, p) .
$$

This is to say that a consumer chooses the durable good that maximizes her expected present value.

\subsection{Allocations within a Period}

Next, we describe durable good allocations within a period. As we have seen in the previous subsection, our notation must capture the joint distribution of durables and consumers both before and after trade. We begin by defining the set of durable good allocations. A durable good allocation is a probability measure $\mu \in \mathcal{M}_{A \times \Omega}$, where $\mu_{A}=\nu{ }^{5}$ That is, a durable good allocation is a joint distribution of durables and consumers with the property that its marginal distribution over consumer tastes is consistent with the exogenous distribution of consumer tastes $\nu$. A point $(a, \omega) \in A \times \Omega$ describes a consumer of type $a$ holding the durable $\omega$ (or no durable, in the case $\omega=\emptyset$ ).

As noted above, an allocation within a period must contain information about both the pre- and post- trade durable good allocations. Therefore we define the collection of allocations within a period to be $\mathcal{T}=\left\{\tau \in \mathcal{M}_{A \times \Omega_{1} \times \Omega_{2}}: \tau_{A}=\nu\right\}$, with $\Omega_{1}=\Omega_{2}=\Omega$. If $\tau \in \mathcal{T}$, it follows immediately that $\tau_{A \times \Omega_{1}}$ and $\tau_{A \times \Omega_{2}}$ are durable good allocations. Thus, we adopt the convention that $\Omega_{1}$ represents the set of states before trade and $\Omega_{2}$ represents the set of states after trade. Of course, the assumption of stationarity imposes constraints on the relationship between these two distributions. In particular, stationarity requires that, (1) if the durable good allocation $\tau_{A \times \Omega_{1}}$ represents initial endowments, $\tau_{A \times \Omega_{2}}$ should represent the durable good allocation after all consumers have made optimizing trades based on those

\footnotetext{
${ }^{5}$ If $E$ is a metric space, then $\mathcal{B}_{E}$ represents the collection of Borel sets on $E$. We denote by $\mathcal{M}_{E}$ the space of all probability measures on $E$. If $\mu \in \mathcal{M}_{E \times F}$, we denote by $\mu_{E}$ the marginal distribution on $E$.
} 
endowments and (2) when $\tau_{A \times \Omega_{2}}$ depreciates, it becomes $\tau_{A \times \Omega_{1}}$. Although depreciation and trade actually occur instantaneously upon entry into each period $t$, the following figure nonetheless approximates the relationship between the timing of events and the durable good allocations $\tau_{A \times \Omega_{1}}$ and $\tau_{A \times \Omega_{2}}$ :

Depreciation Trade

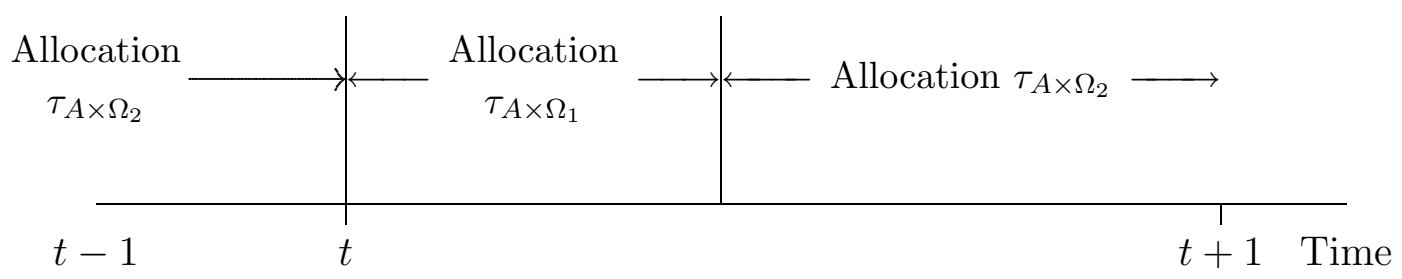

Figure 1

\subsection{Supply Side}

Brand new durables of each type $j$, (i.e., $\omega=(j, 0)$ ) are supplied perfectly elastically at price $\bar{p}_{j}$. There also exists perfectly elastic demand (a scrappage sector) for durables in any state at the scrap price $\underline{p}_{j}<\bar{p}_{j}$. The flow supply correspondence is therefore given by $\eta: P \rightarrow \Re^{\Omega}$ such that

(Case-I)

$$
\eta_{\emptyset}=[0, \infty)
$$

(Case-II) if $\omega=(j, 0)$ for $j \in \mathcal{J}$,

$$
\eta_{j 0}\left(p_{j}\right)= \begin{cases}\infty & p_{j}>\bar{p}_{j}, \\ {[0, \infty)} & p_{j}=\bar{p}_{j}, \\ 0 & p_{j}<\bar{p}_{j},\end{cases}
$$

(Case-III) if $\omega=(j, s)$ for $j \in \mathcal{J}$ and $s>0$,

$$
\eta_{j s}\left(p_{j}\right)= \begin{cases}0 & p_{j}>\underline{p}_{j} \\ (-\infty, 0] & p_{j}=\underline{p}_{j} \\ -\infty & p_{j}<\underline{p}_{j}\end{cases}
$$

The fact that the choice not to own a durable is always available to every consumer is indicated by (Case-I). (Case-II) states that supply of brand new durable goods is perfectly elastic at the exogenous price $\bar{p}_{j}$, while (Case-III) indicates that demand for scrapped durables is also perfectly elastic at the price $\underline{p}_{j}$.

Supply of durables of all types $j$ in states $s>0$ is controlled by the exogenous depreciation process $\delta: \mathcal{M}_{A \times \Omega_{2}} \rightarrow \mathcal{M}_{A \times \Omega_{1}}$. In particular, if $\mu \in \mathcal{M}_{A \times \Omega_{2}}$ is the durable goods allocation 
which prevails before depreciation, then $\mu^{\prime}=\delta(\mu)$ is the durable goods allocation which prevails after depreciation. Because $\Omega$ is finite, we may always represent $\mu$ by a collection of component distributions $\left\{\mu_{\omega}\right\}_{\omega \in \Omega}$. Using this notation, $\delta$ is given by

$$
\delta(\mu)_{\omega}= \begin{cases}\mu_{\omega} & \omega=\emptyset, \\ \sum_{s^{\prime}} \phi_{j}\left(s^{\prime}, s\right) \mu_{j s^{\prime}} & \omega=(j, s) .\end{cases}
$$

The "outside good" (non-ownership of a durable) does not depreciate. For all other durables, the probability of arriving in state $(j, s)$ is simply the sum of the joint probabilities of being in state $\left(j, s^{\prime}\right)$ and making the ( $j$-specific) state transition from $s^{\prime}$ to $s$. It is easily shown (see Fact 2 below) that the depreciation mapping does not disturb $\mu_{A}$, the marginal distribution over consumer types.

\subsection{Stationary Equilibrium}

A stationary equilibrium is a list $\left(\tau^{*}, p^{*}\right) \in \mathcal{T} \times P$ such that

(i) $\tau_{A \times \Omega_{1}}^{*}=\delta\left(\tau_{A \times \Omega_{2}}^{*}\right)$,

(ii) $\tau^{*}\left(\left\{\left(a, \omega, \omega^{\prime}\right) \in A \times \Omega_{1} \times \Omega_{2}: \tilde{V}\left(a, \omega, \omega^{\prime}, p^{*}\right) \geq \tilde{V}\left(a, \omega, \Omega, p^{*}\right)\right\}\right)=1$, and

(iii) $\tau_{\Omega_{1}}^{*}-\tau_{\Omega_{2}}^{*} \in \eta\left(p^{*}\right)$.

Condition (i) states that the pre-trade/endowment durable goods allocation must be feasible relative to the technology for depreciation, $\delta$. Condition (ii) says that almost every consumer chooses optimally at the equilibrium prices $p^{*}$. In particular, the contents of the brace in condition (ii) describes the set of all combinations of agents $(a)$, pre-trade/endowment durables $(\omega)$ and post-trade/replacement durables $\left(\omega^{\prime}\right)$ such that $\omega^{\prime}$ is an optimal choice for agent $a$ given endowment durable $\omega$. The conjunction of Conditions (i) and (ii) embodies the stationarity of the equilibrium. Condition (i) states that the post-trade durable goods allocation $\tau_{A \times \Omega_{2}}^{*}$, which prevails at the end of every period $t$, is transformed via depreciation (at the beginning of period $t+1$ ) into the pre-trade/endowment durable goods allocation $\tau_{A \times \Omega_{1}}^{*}$. Condition (ii) states that the pre-trade/endowment durable goods allocation $\tau_{A \times \Omega_{1}}^{*}$, which prevails at the beginning of every period $t$, is transformed via optimal replacement into the post-trade durable goods allocation $\tau_{A \times \Omega_{2}}^{*}$, which prevails throughout period $t$. Condition (iii) is the market clearing condition.

\subsection{The Existence Theorem}

To prove the existence of equilibrium, we impose the following regularity conditions: 


\section{Assumptions}

A1. There exists $\bar{\beta}$ such that $0 \leq \beta^{a} \leq \bar{\beta}<1$, for any $a \in A$.

A2. For any $a \in A, j \in \mathcal{J}$ and $s<S, \bar{u}_{j} \geq u_{j s}^{a} \geq u_{j s+1}^{a} \geq u_{\emptyset}^{a}$ for some $\bar{u}_{j} \geq 0$. As previously noted, we normalize $u_{\emptyset}^{a}=0$.

A3. There exists $\bar{c}$ such that, for any $a \in A, 0 \leq c^{a} \leq \bar{c}$.

A4. For every $j \in \mathcal{J}$, the transition probability function $\phi_{j}: \mathcal{S} \times \mathcal{S} \rightarrow[0,1]$ satisfies

(i) $\sum_{s^{\prime}=s}^{S} \phi_{j}\left(s, s^{\prime}\right)=1$ for any $s \in \mathcal{S}$,

(ii) $\phi_{j}\left(s, s^{\prime}\right)>0$ for any $s<s^{\prime} \leq S$.

Given these assumptions, we can prove the existence of a stationary equilibrium. Our main result is:

Theorem. Suppose A1-A4 hold. Then there exists a stationary equilibrium in our durable goods economy.

\section{Remarks on Assumptions}

Assumptions A1-A3 are used to compactify the space $\mathcal{T}$ in the topology of weak convergence. Assumptions A3 and A4 are used to show boundedness of the equilibrium prices. Showing boundedness of the equilibrium prices is significant because truncation of the price space to a compact set is central to the application of a fixed point theorem. In establishing bounds on the price space, a difficulty which must be confronted is that value function has prices as arguments. As a result, consumers take capital gains and losses into account when determining their optimal buying and selling plans (e.g., most consumers consider an automobile's resale value before purchasing). Thus, we need to guarantee that prices do not increase without bound by identifying an explicit upper bound. Note that, even under the assumptions on tastes and the depreciation process, prices are not bounded above by $\bar{p}_{j}$ (even though durables in state $(j, 0)$ are supplied perfectly elastically at this price). The following example illustrates this point.

Example. Suppose that there is one type of durable good $(J=1)$ and one type of consumer. Suppose further that $S=\{0,1,2\}$ and the transition probabilities are defined by

$$
\begin{array}{lll}
\phi(0,0)=0 & \phi(0,1)=0.25 & \phi(0,2)=0.75 \\
\phi(1,1)=0.75 & \phi(1,2)=0.25 \\
& \phi(2,2)=1
\end{array}
$$

We let $u_{0}=1, u_{1}=0.9$ and $u_{2}=0$. New and scrap prices are given by $\bar{p}=1$ and $p=0$. The discount rate is $\beta=0.8$. The transaction cost is $c=0$. Then the equilibrium prices are 
given by $p_{0}=\bar{p}=1, p_{1}=1.5$ and $p_{2}=\underline{p}=0$.

The above result is easily demonstrated. Initially, suppose that the optimal decision rule is characterized by keeping any durable in states 0 or 1 and replacing any durable in state 2 with a brand new (state 0) durable. Then, since every consumer follows this decision rule, the value function is given by

$$
\begin{aligned}
& V(0)=1+0.8(0.25 V(1)+0.75 V(2))=1+0.2 V(1)+0.6 V(2) \\
& V(1)=0.9+0.8(0.75 V(1)+0.25 V(2))=0.9+0.6 V(1)+0.2 V(2) \\
& V(2)=\underline{p}-\bar{p}+V(0) \quad=-1+V(0)
\end{aligned}
$$

Solving this matrix equation gives $V(0)=2.5, V(1)=3$ and $V(2)=1.5$, from which the prices given above follow. Notice that $p_{1}>p_{0}$. The intuition behind this result can be easily explained by supposing perfect rental markets for durables in all states $s=0,1,2$. Since more degraded durables provide lower services (i.e., $u_{0}>u_{1}>u_{2}$ ), it is clear that the rental prices of these durables must be decreasing with $s$. Furthermore, since durables in states 0 and 1 provide similar services, their rental prices should be similar. Because rental markets are perfect, the value of holding a durable in perpetuity (i.e., its price) is simply the expected present discounted value of its future rental prices. Now observe that durables occupying state 0 are very likely to make a transition to a low-quality state in the following period, while durables in state 1 are very likely not to degrade at all. Recalling that the rental prices of durables 0 and 1 are similar, durable 1 clearly generates a higher expected future reward and will therefore command a higher market price.

Finally, consider the following modification of Assumption A4 which allows for deterministic depreciation.

A4'. For every $j \in \mathcal{J}$, the transition probability function $\phi_{j}: \mathcal{S} \times \mathcal{S} \rightarrow[0,1]$ is given, for any $s, t \in \mathcal{S}$ with $s<S$, by

$$
\phi_{j}(s, t)= \begin{cases}1 & \text { if } t=s+1 \\ 0 & \text { otherwise }\end{cases}
$$

and $\phi_{j}(S, S)=1$.

Under Assumptions A1-A3 and A4', our Theorem will also hold. The only part of the proof which must be altered is the construction of the upper bound on prices, which will be a different upper bound under Assumption A4'. The argument in the Lemma needs some modification, but the technique of the proof (namely, presenting a replacement policy which dominates that of replacement with the durable which is in excess demand) remains the same. 


\section{The Existence Proof}

The outline of the proof is as follows. First, we show that each consumer's value function exists and is unique and continuous (Fact 1) and that the depreciation mapping defined in (??) is continuous (Fact 2). Second, we construct a correspondence which maps each durable goods allocation within a period into the collection of all allocations within a period which are technologically feasible and represent optimal choices by consumers. Any durable goods allocation within a period in the target space of this correspondence will therefore satisfy the first and second equilibrium conditions. We show that this correspondence is nonempty, convex-valued and has closed graph (Fact 3). Third, we define an aggregate demand correspondence which describes the extent to which the third equilibrium condition is violated and show that this correspondence is also nonempty, convex-valued and has closed graph (Fact 4). Fourth, we construct a Gale-Nikaido type price correspondence (Debreu: 1954, 5.6 (1)) that is nonempty, convex-valued, and has a closed graph (Fact 5). Using these component mappings, we construct a product mapping that (by the Fan-Glicksburg Fixed Point Theorem) has a fixed point (Fact 6). Finally, we show that the fixed point is actually a stationary equilibrium of our economy. Our Lemma shows that the Gale-Nikaido mapping turns out to be useful in our setting, as well.

First, let $\tilde{P} \equiv \prod_{j \in J}\left(\left[\underline{p}_{j}, \bar{p}_{j}\right] \times \prod_{s=1}^{S}\left[\underline{p}_{j}, \bar{M}_{j}\right]\right) \times\{0\}$, where

$$
\bar{M}_{j} \equiv \bar{p}_{j}+\frac{\bar{p}_{j}-\underline{p}_{j}+\bar{c}}{\min _{s>0} \phi_{j}(0, s)} .
$$

Note that this truncation of $P$ is a compact set. Because demand for scrapped durables is perfectly elastic at $\underline{p}_{j}$, an equilibrium price never lies below $\underline{p}_{j}$ for any state. Furthermore, the price of a brand new durable of type $j$ cannot exceed $\bar{p}_{j}$, so $p_{j 0}$ (the price of a type $j$ durable in state 0$)$ must lie in $\left[p_{j}, \bar{p}_{j}\right]$. For other states, we do not know if price is bounded above by $\bar{p}_{j}$, so we set a large upper bound $\bar{M}_{j}$. Assumption A4(ii) guarantees that the denominator of $\bar{M}_{j}$ is positive. The last component, $\{0\}$, is the price of not holding a durable. Using this truncation of $P$, we can show that the value function is well-behaved:

Fact 1. For any $a \in A$, any $p \in \tilde{P}$, and any $\omega \in \Omega, V(a, \omega, p)$ is uniquely determined. Also, $V$ is continuous.

Proof. It is easy to see that Blackwell's sufficient conditions for contraction mapping are satisfied in this case (see Lucas and Stokey (1989)). Thus, the value function exists and is uniquely determined. Continuity of $V$ is apparent.

An immediate consequence of Fact 1 is that $\tilde{V}: A \times \Omega_{1} \times \Omega_{2} \times P \rightarrow \Omega_{+}$is a continuous function. Next, we show that the depreciation process is similarly well-behaved:

Fact 2. If $\delta: \mathcal{M}_{A \times \Omega_{2}} \rightarrow \mathcal{M}_{A \times \Omega_{1}}$ is as given in (??), then $\delta$ is continuous.

Proof: Let $\mu^{n}$ be a sequence of probabilities in $\mathcal{M}_{A \times \Omega_{2}}$ converging to $\mu$ in the topology of weak convergence. Then $\delta$ is continuous if $\delta\left(\mu^{n}\right) \rightarrow \delta(\mu)$ weakly, as well. Let $\left\{\mu_{w}^{n}\right\}_{\omega \in \Omega}$ 
represent the component distributions of $\mu^{n}$ and $\left\{\delta\left(\mu^{n}\right)_{w}\right\}_{\omega \in \Omega}$ those of $\delta\left(\mu^{n}\right)$. Clearly, $\delta\left(\mu^{n}\right) \rightarrow \delta(\mu)$ iff every component distribution converges. If $\omega=\emptyset, \delta\left(\mu^{n}\right)_{\emptyset}=\mu_{\emptyset}^{n}$, so convergence follows immediately. Therefore, let $\omega=(j, s)$ and let $f: A \rightarrow \Re$ be bounded and continuous. We wish to show that $\int_{A} f d\left(\delta\left(\mu^{n}\right)_{j s}\right) \rightarrow \int_{A} f d\left(\delta(\mu)_{j s}\right)$. We have

$$
\begin{aligned}
\int_{A} f d\left(\delta\left(\mu^{n}\right)_{j s}\right) & =\int_{A} f d\left[\sum_{s^{\prime}} \phi_{j}\left(s^{\prime}, s\right) \mu_{j s^{\prime}}^{n}\right], \\
& =\sum_{s^{\prime}} \phi_{j}\left(s^{\prime}, s\right) \int_{A} f d \mu_{j s^{\prime}}^{n}
\end{aligned}
$$

where the second step follows from the Lebesgue-Radon-Nikodym Theorem (Folland, p. 84, Theorem 3.8). Using our hypothesis that $\mu^{n} \rightarrow \mu$ yields the desired result.

Define the correspondences $D: \mathcal{T} \rightarrow \mathcal{T}, B: \tilde{P} \rightarrow \mathcal{T}$ and $\theta: \mathcal{T} \times \tilde{P} \rightarrow \mathcal{T}$ by

$$
\begin{aligned}
D(\tau) & =\left\{\tau^{\prime} \in \mathcal{T}: \tau_{A \times \Omega_{1}}^{\prime}=\delta\left(\tau_{A \times \Omega_{2}}\right)\right\}, \\
B(p) & =\left\{\tau^{\prime} \in \mathcal{T}: \tau^{\prime}\left(\left\{\left(a, \omega, \omega^{\prime}\right) \in A \times \Omega_{1} \times \Omega_{2}: \tilde{V}\left(a, \omega, \omega^{\prime}, p\right) \geq \tilde{V}(a, \omega, \Omega, p)\right\}\right)=1\right\}, \\
\theta(\tau, p) & =D(\tau) \cap B(p) .
\end{aligned}
$$

Given any initial durable goods allocation $\tau, D(\tau)$ gives the collection of all durable goods allocations which are feasible, given the depreciation technology $\delta$. Given any price vector $p$, $B(p)$ describes the set of all durable goods allocations within a period in which almost every consumer is optimizing at prices $p$. Consequently, $\theta(\tau, p)$ characterizes the collection of all durable goods allocations within a period which are both technologically feasible from $\tau$ and in which almost every consumer is optimizing at $p$. The following fact is closely related to Jovanovic and Rosenthal (1988), and the proof is therefore relegated to the Appendix.

Fact 3. The mapping $\theta$ is nonempty, convex-valued, and has closed graph.

Next, let us define the aggregate demand correspondence (supply side is not taken into consideration). Let $Z \equiv[-1,1]^{\Omega}$. Let $\zeta: \mathcal{T} \rightarrow Z$ be such that $\zeta(\tau)=\left(\tau_{\Omega_{2}}-\tau_{\Omega_{1}}\right)$. Obviously, $\zeta$ is a continuous function.

Fact $4 . \zeta$ is a continuous function.

Let $\pi: Z \rightarrow \tilde{P}$ be such that $\pi(z)=\arg \max _{p \in \tilde{P}} p \cdot z$. Note that $p_{\emptyset}=0$ and $p_{\emptyset} z_{\emptyset}=0$. This mapping is used in Gale and Nikaido's lemma (see Debreu (1959)). Note that we cannot use a standard argument to show the excess demand is nonnegative, since Walras' law does not hold in our economy. This is the reason why we introduced $\bar{M}_{j}$. As is well-known, $\pi$ has the following nice property (a direct application of the Maximum Theorem):

Fact 5. $\pi$ is nonempty, convex-valued, and has a closed graph.

Let $\varphi: \mathcal{T} \times Z \times \tilde{P} \rightarrow \mathcal{T} \times Z \times \tilde{P}$ be a product of $\theta: \mathcal{T} \times \tilde{P} \rightarrow \mathcal{T}, \zeta: \mathcal{T} \rightarrow Z$, and $\pi: Z \rightarrow \tilde{P}$. This is our fixed point mapping which is nonempty, convex-valued, and has a 
closed graph. From A1-A3, $\mathcal{T}$ is compact in the topology of weak convergence. The sets $Z$ and $\tilde{P}$ are compact in the metric topology. Therefore, by the Fan-Glicksburg Fixed Point Theorem, we obtain the following:

Fact 6. There exists $\left(\tau^{*}, z^{*}, p^{*}\right) \in \mathcal{T} \times Z \times \tilde{P}$ such that $\left(\tau^{*}, z^{*}, p^{*}\right) \in \varphi\left(\tau^{*}, z^{*}, p^{*}\right)$.

In the rest of the proof, we show $\left(\tau^{*}, z^{*}, p^{*}\right) \in \mathcal{T} \times Z \times \tilde{P}$ is a stationary equilibrium. Since $p^{*} \in \pi\left(z^{*}\right)$, we have (i) $z_{j 0}^{*}>0$ implies $p_{j s}^{*}=\bar{p}_{j}$ for any $j \in \mathcal{J}$, (ii) $z_{j s}^{*}>0$ implies $p_{j s}^{*}=\bar{M}_{j}$ for any $j \in \mathcal{J}$ and any $s \in \mathcal{S} \backslash\{0\}$, and (iii) $z_{j s}^{*}<0$ implies $p_{j s}^{*}=\underline{p}_{j}$ for any $j \in \mathcal{J}$ and $s \in \mathcal{S}$. If case (ii) occurs, then $z^{*}$ is not a feasible allocation. The following lemma excludes this case:

Lemma. Under Assumptions $A 3$ and $A 4, z_{j s}^{*} \leq 0$ for any $j \in \mathcal{J}$ and any $s \in \mathcal{S} \backslash\{0\}$.

Proof. Suppose that $z_{j s}^{*}>0$. Then, by the construction of $\pi, p_{j s}^{*}=\bar{M}_{j}$ holds. Since $z_{j s}^{*}>0$, there exists a measurable set $E \subset A$ with $\nu(E)>0$ such that each consumer $a \in E$ chooses $\omega^{\prime}=(j, s)$ as her optimal choice at the prices $p^{*}$. With $a \in E$, and given quasi-linear utility, $a^{\prime}$ 's optimal choice $\omega^{\prime}=(j, s)$ is independent of her current durable goods holdings $\omega \in \Omega$, as long as she transacts in the market. Using this observation, her value function is given by $V\left(a, j, s, p^{*}\right) \equiv u_{j s}^{a}+\beta^{a} \sum_{\tilde{s}=s}^{S} \phi_{j}(s, \tilde{s}) V\left(a, j, \tilde{s}, p^{*}\right)$, where

$$
\begin{aligned}
V\left(a, j, \tilde{s}, p^{*}\right) & =\max \left\{u_{j \tilde{s}}^{a}+\beta^{a} \sum_{s^{\prime}=\tilde{s}}^{S} \phi_{j}\left(\tilde{s}, s^{\prime}\right) V\left(a, j, s^{\prime}, p^{*}\right), V\left(a, j, s, p^{*}\right)+p_{j \tilde{s}}^{*}-p_{j s}^{*}-c^{a}\right\} \\
& \leq \max \left\{u_{j \tilde{s}}^{a}+\beta^{a} \sum_{s^{\prime}=\tilde{s}}^{S} \phi_{j}\left(\tilde{s}, s^{\prime}\right) V\left(a, j, s^{\prime}, p^{*}\right), V\left(a, j, s, p^{*}\right)\right\} \\
& =V\left(a, j, s, p^{*}\right) .
\end{aligned}
$$

The second line follows from $p_{j s}^{*}=\bar{M}_{j} \geq p_{j \tilde{s}}^{*}$, and the third line follows from the fact that $(j, s)$ is an optimal choice in the presence of transaction costs. ${ }^{6}$ Thus, we can conclude $V\left(a, j, s, p^{*}\right) \leq \frac{1}{1-\beta^{a}} u_{j s}^{a} \cdot{ }^{7}$

Now suppose, instead, that consumer $a \in E$ follows a replacement rule in which she always replaces with the durable $\omega^{\prime}=(j, 0)$ and replaces her durable whenever it depreciates to state $(j, s)$ with $s>0$. The present value of following this replacement rule, $\hat{V}\left(a, j, 0, p^{*}\right)$,

\footnotetext{
${ }^{6}$ If transaction costs are zero, $(j, s)$ continues to be an optimal choice, although (??) will hold with equality.

${ }^{7}$ This is an intuitive result. Since $p_{j s}^{*}=\bar{M}_{j}$, the consumer $a \in E$ cannot expect capital gains. Hence, her value function is bounded above by the discounted value of her consumption stream only.
} 
is

$$
\begin{aligned}
& \hat{V}\left(a, j, 0, p^{*}\right)=u_{j 0}^{a}+\beta^{a}\left[\phi_{j}(0,0) \hat{V}\left(a, j, 0, p^{*}\right)\right. \\
&\left.\quad+\sum_{\tilde{s}=1}^{S} \phi_{j}(0, \tilde{s})\left(\hat{V}\left(a, j, 0, p^{*}\right)+\left(p_{j \tilde{s}}^{*}-\bar{p}_{j}\right)-c^{a}\right)\right] \\
&=u_{j 0}^{a}+\beta^{a} \hat{V}\left(a, j, 0, p^{*}\right)+\beta^{a} \sum_{\tilde{s}=1}^{S} \phi_{j}(0, \tilde{s})\left(p_{j \tilde{s}}^{*}-\bar{p}_{j}-c^{a}\right) \\
&=u_{j 0}^{a}+\beta^{a} \hat{V}\left(a, j, 0, p^{*}\right)+\beta^{a} \phi_{j}(0, s)\left(\bar{M}_{j}-\bar{p}_{j}-c^{a}\right) \\
& \quad+\beta^{a} \sum_{\tilde{s} \in \mathcal{S} \backslash\{0, s\}} \phi_{j}(0, \tilde{s})\left(p_{j \tilde{s}}^{*}-\bar{p}_{j}-c^{a}\right) \\
& \geq u_{j 0}^{a}+\beta^{a} \hat{V}\left(a, j, 0, p^{*}\right)+\beta^{a} \phi_{j}(0, s)\left(\bar{M}_{j}-\bar{p}_{j}-c^{a}\right) \\
& \quad+\beta^{a} \sum_{\tilde{s} \in \mathcal{S} \backslash\{0, s\}} \phi_{j}(0, \tilde{s})\left(\underline{p}_{j}-\bar{p}_{j}-c^{a}\right)
\end{aligned}
$$

The last inequality follows from the definition of $\bar{M}_{j}$. This implies that $\hat{V}\left(a, j, 0, p^{*}\right)>$ $\frac{1}{1-\beta^{a}} u_{j 0}^{a} \geq \frac{1}{1-\beta^{a}} u_{j s}^{a} \geq V\left(a, j, s, p^{*}\right)$. Thus, $(j, s)$ could not have been an optimal choice for any consumer $a \in E$, a contradiction. It follows that $z_{j s}^{*} \leq 0$ for any $j \in \mathcal{J}$ and $s>0$.

By our Lemma, case (ii) is impossible at a fixed point of $\varphi$, so $z_{j s}^{*}>0$ only if $s=0$. To complete the proof of the theorem, let $y^{*}=-z^{*}$. Apparently, $y^{*} \in \eta\left(p^{*}\right)$, so $\left(\tau^{*}, y^{*}, p^{*}\right)$ is a stationary equilibrium. 


\section{Appendix}

This section provides a proof of Fact 3. To do so, it is first necessary to prove two facts, $3 \mathrm{a}$ and $3 \mathrm{~b}$, related (respectively) to the mappings $D(\cdot)$ and $B(\cdot)$.

Fact 3a. D is nonempty, convex-valued and has closed graph.

Proof. We first show that $D$ is nonempty. Begin by choosing $\tau \in \mathcal{T}$, and let $\tau_{A \times \Omega_{1}}^{\prime}=$ $\delta\left(\tau_{A \times \Omega_{2}}\right)$. Let $\left\{\tau_{A \times \Omega_{1}(\omega)}^{\prime}\right\}_{\omega \in \Omega_{1}}$ represent the collection of component measures for $\tau_{A \times \Omega_{1}}^{\prime}$ (similarly for $\tau$ ). By definition of the marginal,

$$
\begin{aligned}
\tau_{A}^{\prime} & =\sum_{\omega \in \Omega_{1}} \tau_{A \times \Omega_{1}(\omega)}^{\prime} \\
& =\tau_{A \times \Omega_{2}(\emptyset)}+\sum_{j} \sum_{s} \sum_{s^{\prime}} \phi_{j}\left(s^{\prime}, s\right) \tau_{A \times \Omega_{2}\left(j s^{\prime}\right)} \\
& =\tau_{A \times \Omega_{2}(\emptyset)}+\sum_{j} \sum_{s^{\prime}} \tau_{A \times \Omega_{2}\left(j s^{\prime}\right)} \\
& =\sum_{\omega \in \Omega_{2}} \tau_{A \times \Omega_{2}(\omega)}
\end{aligned}
$$

which is $\nu$ because $\tau \in \mathcal{T}$. Since $\tau_{A \times \Omega_{1}}^{\prime}=\delta\left(\tau_{A \times \Omega_{2}}\right)$ and $\tau_{A}^{\prime}=\nu$, it follows that $\tau^{\prime} \in D(\tau)$.

Next we show that $D$ has closed graph. Let $\left(\tau^{n}, \sigma^{n}\right)$ be a sequence in $\mathcal{T} \times \mathcal{T}$ with $\left(\tau^{n}, \sigma^{n}\right) \rightarrow(\tau, \sigma)$ and $\sigma^{n} \in D\left(\tau^{n}\right)$. Then it follows that $D$ has closed graph if $\sigma \in D(\tau)$. Since $\sigma^{n} \in D\left(\tau^{n}\right), \sigma_{A \times \Omega_{1}}^{n}=\delta\left(\tau_{A \times \Omega_{2}}^{n}\right)$. Consequently, we have $\int f d \sigma_{A \times \Omega_{1}}^{n}=\int f d \delta\left(\tau_{A \times \Omega_{2}}^{n}\right)$ for every bounded, uniformly continuous function $f: A \times \Omega \rightarrow \Re$. It follows that, for any $n$,

$$
\begin{aligned}
\mid \int f d \sigma_{A \times \Omega_{1}} & -\int f d \delta\left(\tau_{A \times \Omega_{2}}\right) \mid \\
& =\left|\int f d \sigma_{A \times \Omega_{1}}-\int f d \sigma_{A \times \Omega_{1}}^{n}+\int f d \delta\left(\tau_{A \times \Omega_{2}}^{n}\right)-\int f d \delta\left(\tau_{A \times \Omega_{2}}\right)\right| \\
& \leq\left|\int f d \sigma_{A \times \Omega_{1}}-\int f d \sigma_{A \times \Omega_{1}}^{n}\right|+\left|\int f d \delta\left(\tau_{A \times \Omega_{2}}^{n}\right)-\int f d \delta\left(\tau_{A \times \Omega_{2}}\right)\right| .
\end{aligned}
$$

Proceeding to the limit, and using Fact 2, $\int f d \sigma_{A \times \Omega_{1}}=\int f d \delta\left(\tau_{A \times \Omega_{2}}\right)$ for every bounded, uniformly continuous $f$, so that (Parthasarathy, p. 39, Theorem 5.9) $\sigma_{A \times \Omega_{1}}=\delta\left(\tau_{A \times \Omega_{2}}\right.$ ), and the result follows. Convex-valuedness is immediate.

Fact 3 b. B is nonempty, convex-valued and has closed graph.

Proof. By the Maximum Theorem (Berge, p. 116), $\tilde{V}^{*}(a, \omega, p)=\max _{\omega^{\prime} \in \Omega} \tilde{V}\left(a, \omega, \omega^{\prime}, p\right)$ is continuous and $M(p) \equiv\left\{\left(a, \omega, \omega^{\prime}\right) \in A \times \Omega_{1} \times \Omega_{2}: \tilde{V}\left(a, \omega, \omega^{\prime}, p\right)-\tilde{V}^{*}(a, \omega, p) \geq 0\right\}$ is measurable (and nonempty). It follows that there exists a probability whose support is $M(p)$, so $B(p)$ is nonempty. Convex-valuedness is immediate. 
Finally, we show that $B$ has closed graph. Let $\left(p^{n}, \tau^{n}\right)$ be a sequence in $\tilde{P} \times \mathcal{T}$ with $\left(p^{n}, \tau^{n}\right) \rightarrow(p, \tau)$ and $\tau^{n} \in B\left(p^{n}\right)$ for every $n$. Then $B$ has closed graph if $\tau \in B(p)$, so it suffices to show that $\tau(M(p))=1$. Let $\operatorname{supp}(\tau)$ denote the support of $\tau{ }^{8}$ We then have the following chain of inclusions

$$
\operatorname{supp}(\tau) \subset \operatorname{Ls} \operatorname{supp}\left(\tau^{n}\right) \subset \operatorname{Ls} M\left(p^{n}\right) \subset M(p),
$$

where the first inclusion follows from the fact that $\tau^{n} \rightarrow \tau$ weakly, the second follows from the fact that $\tau^{n} \in B\left(p^{n}\right)$ for every $n$ and the third from the fact that $M(\cdot)$ is upper semicontinuous. Since $\tau$ is a probability, it follows that $\tau(M(p))=1$.

Fact 3. The mapping $\theta$ is nonempty, convex-valued, and has closed graph.

Proof. Using Facts $3 \mathrm{a}$ and $3 \mathrm{~b}$, the latter two properties are immediate, so we need argue only that the intersection is nonempty. Let $\hat{\tau} \in D(\tau)$ and let $\tau^{\prime} \in \mathcal{T}$ be constructed (as above) so as to agree with $\hat{\tau}$ on its $A \times \Omega_{1}$ marginal. For every $p \in \tilde{P}$, let $\sigma_{p}(a, \omega)$ be a measurable selection from the correspondence $\Gamma_{p}: A \times \Omega_{1} \rightarrow \Omega_{2}$ given by

$$
\Gamma_{p}(a, \omega)=\arg \max _{\omega^{\prime} \in \Omega_{2}} \tilde{V}\left(a, \omega, \omega^{\prime}, p\right) .
$$

For each $E \in \mathcal{B}_{A \times \Omega_{1}}$, define

$$
\tau^{\prime}\left(\left\{\left(a, \omega, \omega^{\prime}\right) \mid(a, \omega) \in E, \omega^{\prime}=\sigma_{p}(a, \omega)\right\}\right)=\hat{\tau}_{A \times \Omega_{1}}(E)
$$

Since, if $\sigma_{p}$ has unit measure under $\tau^{\prime}, \tau^{\prime}$ is necessarily in $B(p)$, the proof is complete.

\footnotetext{
${ }^{8}$ The support of a measure $\mu$ is defined to be the complement of the union of all open sets of zero $\mu$-measure. If $\mu$ is a probability, then clearly $\mu(\operatorname{supp}(\mu))=1$.
} 


\section{References}

[1] Akerlof, G., "The Market For Lemons: Qualitative Uncertainty and the Market Mechanism," Quarterly Journal of Economics, 89 (1970), 488-500.

[2] Anderson, S. and V. Ginsburgh, "Price Discrimination via Second-Hand Markets," European Economic Review, 38 (1994), 23-44.

[3] Berge, C., Topological Spaces, Macmillan, New York, 1963.

[4] Berkovec, J., "New Car Sales and Used Car Stocks: A Model of the Automobile Market," RAND Journal of Economics, 16 (1985), 195-214.

[5] Berry, S., J. Levinsohn and A. Pakes, "Automobile Prices in Market Equilibrium," Econometrica, 63 (1995), 841-890.

[6] Bond, E., "Trade in Used Equipment with Heterogeneous Firms," Journal of Political Economy, 91 (1983), 688-705.

[7] Bresnahan, T., "Departures from Marginal-Cost Pricing in the American Automobile Industry: Estimates for 1977-1978," Journal of Econometrics, 17 (1981), 201-227.

[8] Debreu, G., The Theory of Value, Wiley, New York, 1954.

[9] Folland, G., Real Analysis, Wiley, New York, 1984.

[10] Hart, S., W. Hildenbrand and E. Kohlberg, "On Equilibrium Allocations as Distributions on the Commodity Space," Journal of Mathematical Economics, 1 (1974), 159-167.

[11] Hendel, I. and A. Lizzeri, "Adverse Selection in Durable Goods Markets," NBER Working Paper No. 6194, 1997.

[12] Hendel, I. and A. Lizzeri, "Interfering with Secondary Markets," RAND Journal of Economics, 30 (1999), 1-21.

[13] Hildenbrand, W. Core and Equilibria of a Large Economy, Princeton University Press, New Jersey, 1974.

[14] Jovanovic, B. and R.W. Rosenthal, "Anonymous Sequential Games," Journal of Mathematical Economics, 17 (1988), 77-87.

[15] Judd, K., "The Law of Large Numbers with a Continuum of Random Variables," Journal od Economic Theory, 35 (1985), 19-25.

[16] Liebowitz, S., "Durability, Market Structure and New-Used Goods Models," American Economic Review, 72 (1982), 816-824. 
[17] Manski, C., "Equilibrium in Secondhand Markets," Mimeo, Hebrew University of Jerusalem, 1982.

[18] Manski, C., "Analysis of Equilibrium Automobile Holdings in Israel with Aggregate Discrete Choice Models," Transportation Research, 17B (1983), 373-389.

[19] Mas-Colell, A., "A Model of Equilibrium with Differentiated Commodities," Journal of Mathematical Economics, 2 (1975), 263-295.

[20] Mas-Colell, A., "On a Theorem of Schmeidler," Journal of Mathematical Economics, 13 (1984), 201-206.

[21] Munkres, J., Topology, Prentice Hall, New Jersey, 1975.

[22] Parthasarathy, K., Probability Measures on Metric Spaces, Academic Press, New York, 1967.

[23] Rust, J., "Stationary Equilibrium in a Market for Durable Assets," Econometrica, 53 (1985), 783-805.

[24] Sandfort, M.T., "A Dynamic Structural Analysis of Consumer Demand for Automobiles in Sydney, Australia, 1981-1985," Working Paper, 1999.

[25] Sandfort, M.T., "Transaction Costs and Stationary Equilibrium in the Markets for New and Used Durable Goods," Working Paper, 1999.

[26] Sandfort, M.T., "Stationary Equilibrium in New and Used Markets for Durables with Depreciable and Non-Depreciable Components," Working Paper, 1999.

[27] Stokey, N.L., and R.E. Lucas Jr., Recursive Methods in Economic Dynamics, Harvard, Cambridge, 1989.

[28] Swan, P.L., "Durability of Consumer Goods," American Economic Review, 60 (1970), 884-894. 